\title{
Observación: la importancia del arte en la medicina
}

\author{
Pablo Hofmann Salcedo
}

\section{Resumen}

A lo largo de los años, me he enfrentado a momentos de desconcierto, principalmente al escuchar a algunos médicos, generales y especialistas, comentar que Embriología es únicamente una "materia filtro" del plan de estudios. Esos momentos han sido motivo de reflexión y como resultado he intentado atender la necesidad de generar competencias en los estudiantes de primer año de la Licenciatura de Médico Cirujano en la Universidad Nacional Autónoma de México (UNAm); para ello he recurrido al uso de arte pictórico con el fin de favorecer el desarrollo de la capacidad de observación.

El estudio secuencial de las obras pictóricas se realiza mediante el diálogo con y entre los estudiantes, mediante preguntas detonadoras. El beneficio para la formación de médicos radica en que, además de fortalecer y afinar la capacidad de observación, los médicos en formación se sensibilizan y se hacen más empáticos con sus pacientes. Aunque la incorporación del arte visual ha sido una de las estrategias con las que he experimentado, como resultado de la reflexión, ha hecho falta evaluar la eficacia en el logro de las bondades descritas por distintos autores con el uso de estas dinámicas de enseñanza en los grupos de estudiantes a mi cargo. Es necesario, entonces, diseñar instrumentos de evaluación pertinentes para llevar a cabo la comparación con grupos de estudiantes que hayan revisado los contenidos de la asignatura de una manera tradicional.

Palabras clave: arte y educación; educación más alta; conceptos de embriología; competencia de observación.

\section{OBSERVATION: THE IMPORTANCE OF ART IN MEDICINE}

\begin{abstract}
Through the years as a lecturer, I have had puzzling times, mainly when I listened physicians, general practitioners, as well as specialists, express that Embryology is only a filtering course in basic sciences curriculum. Those moments have been the motivation for reflection and, as result, I have tried to address the need of competency development in first year medical students at the National Autonomous University of Mexico (UNAM). For this, I have resorted to the use of pictorial art, in order to stimulate the development of observational skills.

The sequential analysis of the artwork pieces is carried out establishing a dialogue with, and amongst, the students through provocative questions. The formative benefits of these exercises, aside from strengthening and sharpening their observational skills, lie on the sensitization and empathy development. Although the incorporation of visual arts has been one of the strategies that I have experimented with, as result of my reflection process, it is still necessary to assess the efficacy in the attainment of the benefits described by different authors, after the implementation of these class activities. In order to have a measure of the development of these skills, the design of pertinent assessment tools for comparison with students who have studied the subject contents in a more traditional fashion is necessary.
\end{abstract}

Keywords: art and education; higher education; embryology concepts; observation competency.

Dol: http://doi.org/10.22201/codeic.16076079e.2019.v20n6.a7 
Pablo Hofmann Salcedo

https://orcid.org/0000-0002-4701-5117 phofmann@unam.mx

Biólogo, Maestro y Doctor en Ciencias. Jefe de Departamento de Planes y Programas de Estudio de la CODEIC, UNAM.

Mauritshuis online catalogue (s.f). Rembrandt - The Anatomy Lesson of Dr Nicolaes Tulp [fotografía]. Recuperada de: https://commons.wikimedia. org/wiki/File:Rembrandt The Anatomy Lesson of $\mathrm{Dr}$ Nicolaes Tulp.jpg
Con una trayectoria de casi tres décadas como docente en la formación de médicos generales, en el área que constituye las bases científicas para la comprensión de la estructura y función del cuerpo humano he participado en la impartición de las asignaturas de Embriología Humana, Embriología y Genética, Biología del Desarrollo, así como Bioquímica y Biología Molecular. Este escrito es un relato testimonial de los motivos y las intenciones por los cuales he llegado a incorporar el arte pictórico como parte de las actividades de enseñanzaaprendizaje en la asignatura de Embriología Humana, ubicada dentro del plan de estudios de Médico Cirujano en la Facultad de Medicina de la unAm.

A lo largo de los años, me he enfrentado a momentos de desconcierto, principalmente al escuchar a algunos médicos, generales y especialistas,

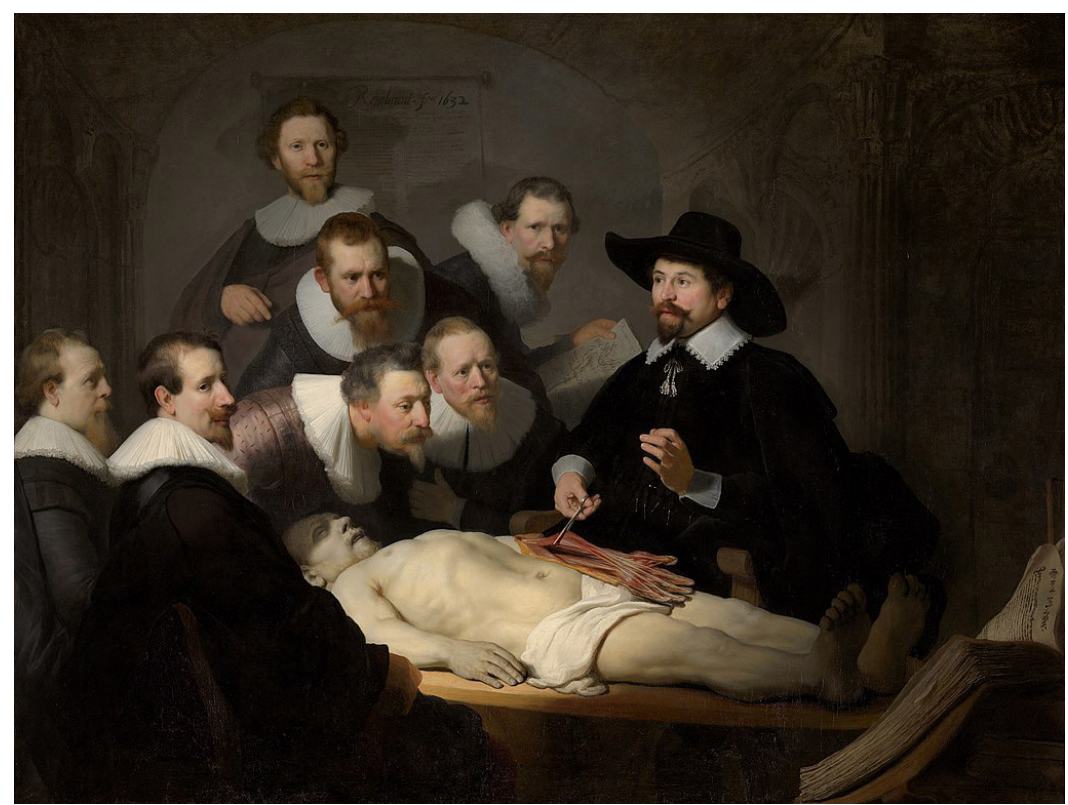
comentar que la Embriología es únicamente una "materia filtro" del plan de estudios. También, cuando los estudiantes han expresado dificultad para entender los conceptos del desarrollo humano, además de su relevancia dentro de la práctica clínica. Esos momentos han sido motivo de reflexión, con la que he cuestionado el qué y el cómo de los procesos del desarrollo que deben aprender los médicos generales en formación, sobre todo para la construcción de su razonamiento crítico y juicio clínico.

Partiendo de la base de que un médico difiere significativamente de un técnico de la salud, se ha considerado necesaria una fase de formación dentro de las ciencias naturales para la construcción del conocimiento que 
Sin autor (s.f). De anatomische les van Dr. Frederick Ruysch [fotografía]. Recuperada de: https://commons.wikimedia.org/ wiki/File:De anatomische les van Dr. Frederick Ruysch.jpg fundamenta el ejercicio clínico y el desarrollo de razonamiento analítico. Posteriormente, se requiere una fase formativa en el ámbito del ejercicio clínico dentro de un entorno hospitalario, bajo la tutoría de un médico competente que, además de dar atención a pacientes, realice investigación (Flexner, 1910).

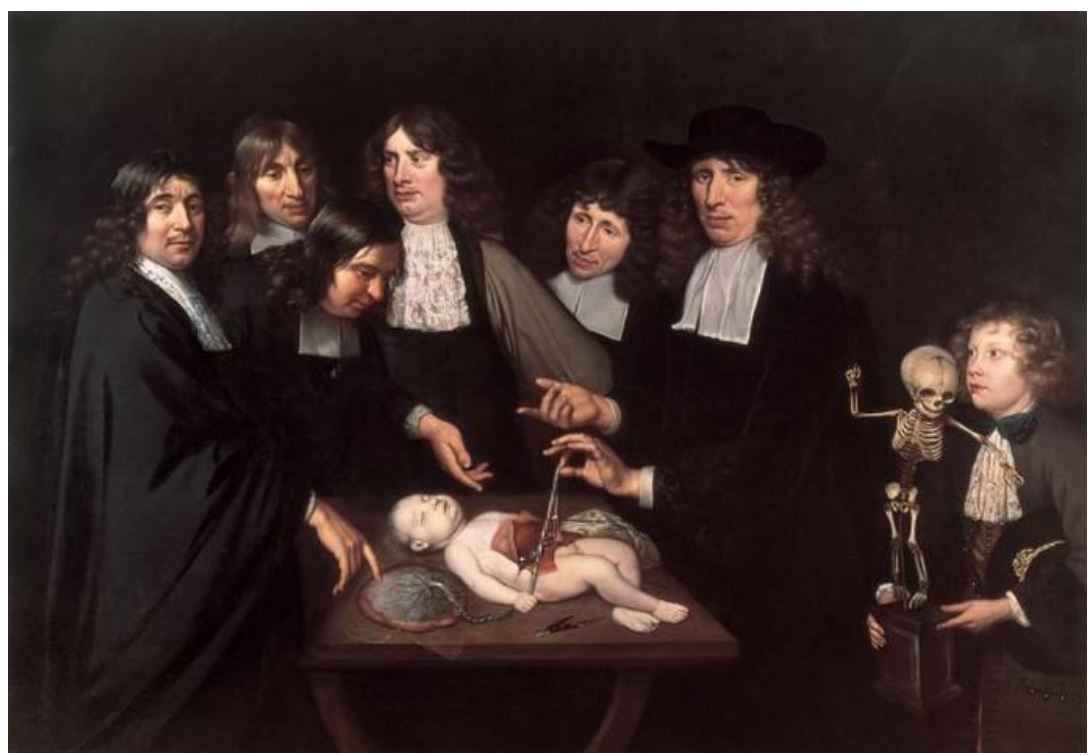

Desde la publicación del reporte para la Fundación Carnegie para el Avance de la Enseñanza (Flexner, 1910), durante el siglo xx la mayoría de las instituciones de educación médica en Norteamérica han estructurado sus currículos con la fórmula general de $2+2$ (dos años de ciencias básicas y dos años de formación clínica). Sin embargo, desde su implementación, ha sido difícil empatar los conceptos científicos fundamentales revisados en la primera fase de formación con su relevancia y aplicación en la atención a la salud, que se desarrolla durante la segunda etapa educativa (Spencer, Brosenitsch, Levine y Kanter, 2008).

Considerando que, a diferencia de un técnico de la salud, entrenado para aplicar protocolos y administrar procedimientos rutinarios con resultados predecibles, un médico con formación científica, además de abordar los problemas clínicos complejos, aplicando conceptos de la biología humana para analizar, relacionar e interpretar signos y síntomas, debe ser capaz de desarrollar, evaluar y modificar los protocolos de atención a la salud. Una de las competencias fundamentales, para atender pacientes en condiciones de salud complejas, es la habilidad para observar y relacionar lo observado con el conocimiento de la estructura y función normal del cuerpo humano. De esta manera, podrá integrar datos y proponer procedimientos de atención en aras de incidir positivamente en la calidad de vida de los pacientes.

Si una competencia se define como el conjunto de conocimientos, habilidades y actitudes, dentro de un contexto de valores éticos, entonces, es necesario desarrollar en el estudiante la capacidad para observar y relacionar el 
conjunto de signos de un problema clínico, con los aspectos científicos de relevancia para la solución del mismo. Por lo tanto, una de las tareas docentes de mayor trascendencia es el diseño de estrategias, para que el médico en formación desarrolle la capacidad de observación e interpretación de datos elementales en los conceptos de las ciencias básicas (fundamentales), con la intención de que pueda proponer las acciones pertinentes en beneficio de sus pacientes.

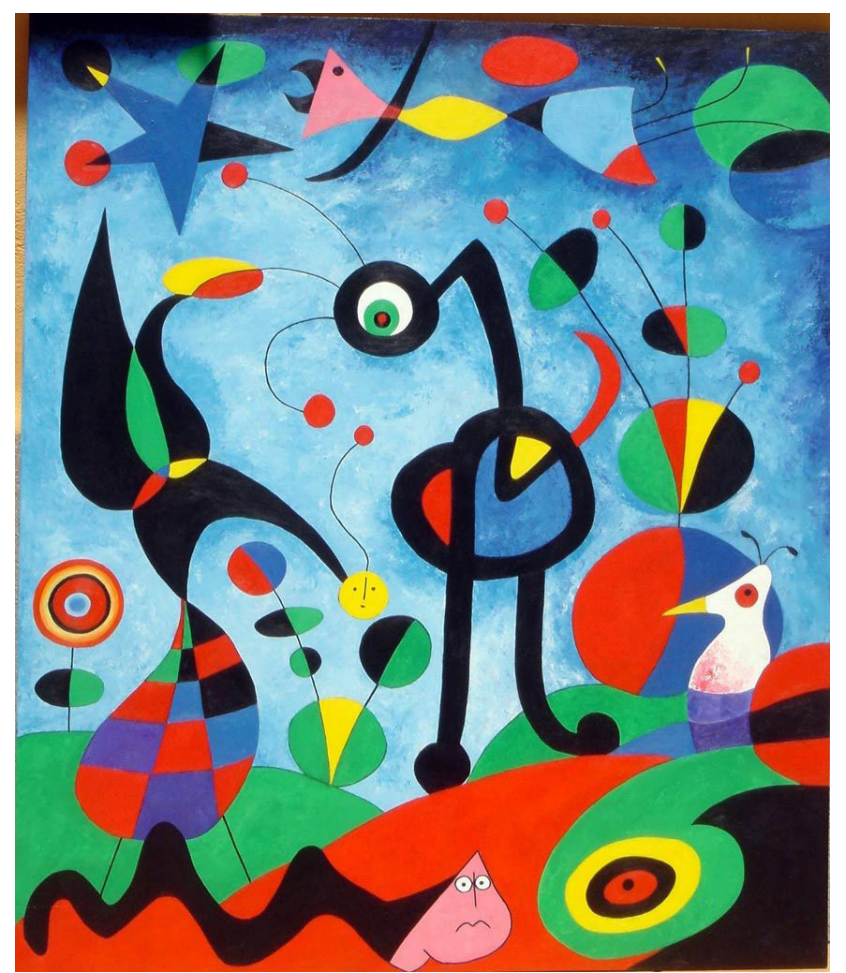

En términos de los beneficios para la formación de médicos, con la incorporación de ejercicios de observación de obras de arte, principalmente pictórico, se ha documentado que además de fortalecer y afinar la capacidad de observación, los médicos en formación se sensibilizan y se hacen más empáticos con sus pacientes (Dolev, Krohner Friedlaender y Braverman, 2001; Jones, 2014; Shapiro y Shallit, 2014). Con el ejercicio constante para el desarrollo y fortalecimiento de la empatía, el ejercicio de la profesión se ve beneficiado en términos humanísticos, situación que sigue siendo un problema pobremente atendido en la educación médica de fórmula 2 + 2 tradicional (Flexner, 1925; Cooke, Irby, Sullivan y Ludmerer, 2006; Jones, 2014).

Como resultado de mi proceso de reflexión he intentando atender la necesidad de generar competencias en los estudiantes del primer año de la licenciatura de Médico Cirujano en la UNAm. Así, he recurrido al uso de arte pictórico para favorecer el desarrollo de la capacidad de observación, antes

Humberto Carrillo Pineda (2015). Joan Miro. Recuperado de: https://humbertocarrillopineda. tumblr.com/ post/132184053267/joan-miro de iniciar la revisión de los contenidos de la asignatura que actualmente se denomina Embriología Humana, dentro del currículo de la licenciatura en la Facultad de Medicina.

En relación con los cuadros, que se analizan secuencialmente durante el desarrollo de las primeras sesiones de clase, se establece el diálogo con y entre los estudiantes mediante preguntas detonadoras respecto a qué es lo que perciben en la imagen, tratando de obtener información con la mayor cantidad de detalle posible (escena general, personaje o personajes representados, posible contexto —cronológico, científico, social o educativo-, actividad representada, actitud o actitudes de los personajes, iluminación, etcétera). En seguida de la discusión general para cada obra, se hace referencia al mapa curricular de la licenciatura (Médico Cirujano, disponible en el sitio electrónico de la facultad), para que indiquen la o las asignaturas con las que se relaciona la pintura y argumenten dicha interpretación. De la misma manera, se promueve la reflexión y explicación de la relación de los elementos de cada una de las escenas con la asignatura de Embriología Humana. Un caso de interés, es que, en repetidas ocasiones, cuando se llega al análisis de la obra de Rob Gonsalves, 
The Sun Sets Sail, los estudiantes relacionan la composición y sus elementos con la asignatura de Embriología, pese a que no contenga explícitamente alguna alusión a conceptos de las ciencias básicas de la medicina.

En el campo de acción de la Embriología Humana, los contenidos de la asignatura se centran principalmente en la descripción de fenómenos del desarrollo, que son complejos y multidimensionales, además de involucrar fases y períodos críticos. Los efectos permanentes de los procesos del desarrollo humano, tanto normales como con fallas en su regulación, son la parte medular, y su conocimiento es de relevancia para su aplicación en el ámbito clínico.

Muchos son los factores que pueden alterar la regulación normal del desarrollo. Entre ellos se encuentran las características genéticas de los progenitores, el estado nutricional y de salud general de la madre (que gesta al

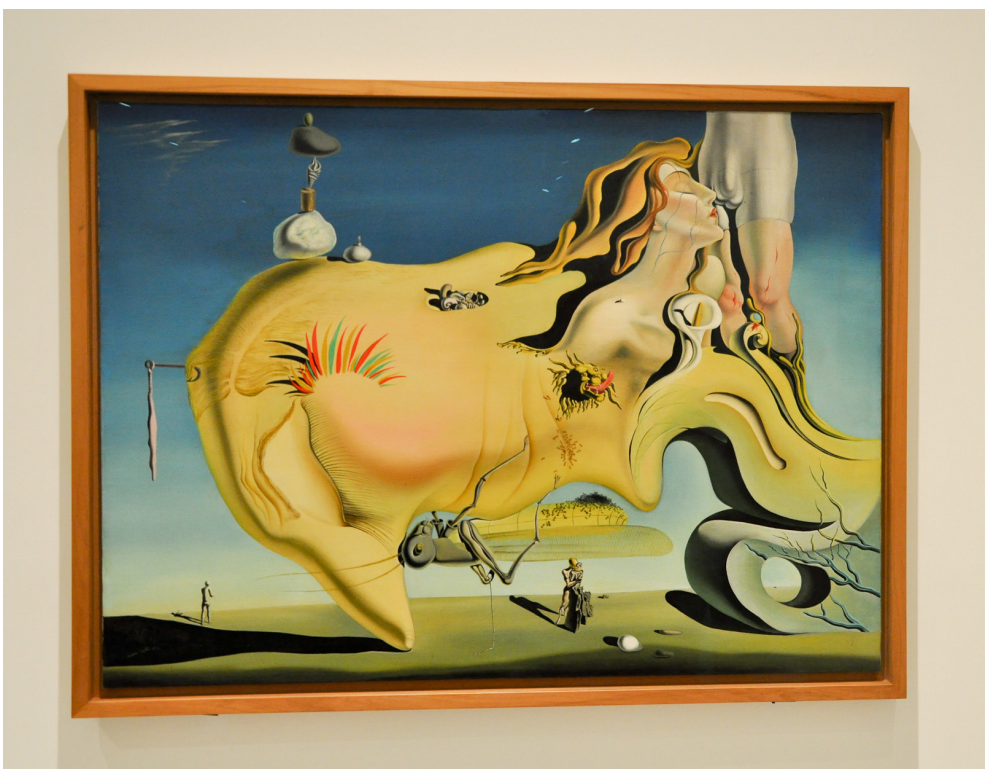
producto en formación), su exposición a factores nocivos ambientales, así como procedimientos diagnósticos y terapéuticos que podrían ser necesarios para la mujer gestante. En caso de que un médico dé atención a una mujer en edad reproductiva, es una responsabilidad ética tener conocimiento de la posibilidad de un embarazo $y$, si este es el caso, la etapa en la que éste se encuentra. Con conocimiento pleno de la fisiología del embarazo y las bases biológicas del desarrollo, el médico podrá considerar todos los factores para la toma de decisiones, mediante un razonamiento crítico, respecto a procedimientos diagnósticos y terapéuticos que resulten en el máximo beneficio para la mujer y su producto. En caso de que el motivo de atención sea el embarazo mismo, el médico deberá ser capaz de comunicar claramente

Nico_ (2011). Salvador Dalí: Rostro del gran masturbador [fotografía]. Recuperada de https://www.flickr.com/ photos/nico /6887018056/in/ photostream/ a la paciente, de acuerdo con su nivel comprensión y considerando la posible respuesta emocional, los aspectos científicos que subyacen a su condición (Association of American Medical Colleges - Howard Hughes Medical Institute [AAMC-HHMI] Committee, 2009).

Para abordar los contenidos de la asignatura, al menos de manera inicial, y facilitar la identificación de los cambios anatómicos durante el desarrollo, he utilizado la comparación de pares secuenciales de imágenes que promueve la comprensión, abstracción y aprendizaje (Gentner, 2010; Kurtz, Boukrina y Gentner, 2013; Goldwater y Gentner, 2015; Ferry, Hespo y Gentner, 2015), aun cuando el lenguaje específico para el campo disciplinario no es del dominio de los estudiantes.

A manera de introducción a la estrategia de comparación de imágenes y como etapa transitoria a los contenidos propios de la asignatura, he utilizado arte pictórico nuevamente. Este ejercicio consiste en la comparación de obras 


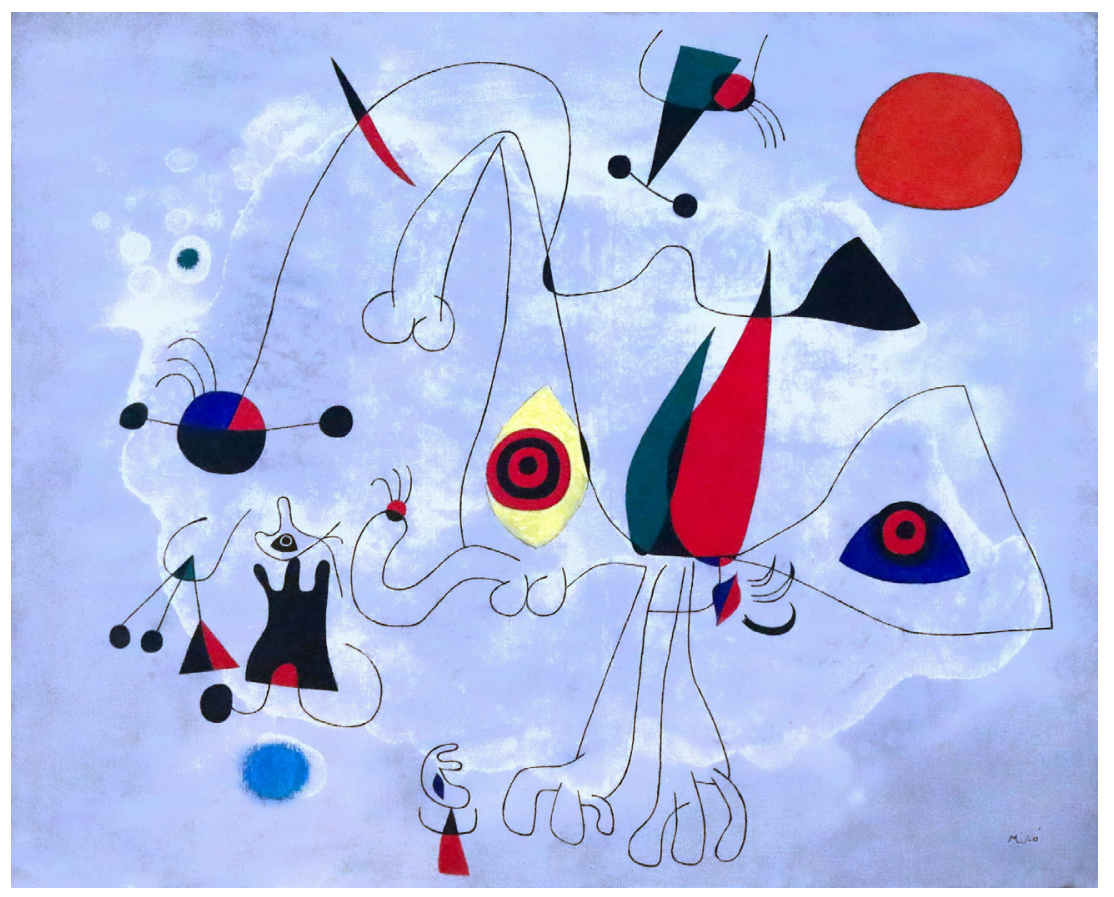

Jean Louis Mazieres (2016). IMG_5758 Joan Miro. 1893-

1983. Femme et oiseaux au lever du soleil. Woman and Birds at sunrise. 1946. Fondation Joan Miro. Barcelone [fotografía]. Recuperada de: https://www.flickr.com/photos/ mazanto/31858511361 de dos artistas y estilos distintos, con el mismo concepto escénico, en el cual los estudiantes deben identificar el tema general de ambas obras, los estilos o escuelas a las que pertenecen, además de encontrar, por lo menos, tres similitudes y tres diferencias entre ellas.

Una vez que se ha realizado la comparación de los dos cuadros, se abordan los contenidos generales de la materia de Embriología con la revisión de los cambios estructurales en las etapas embrionarias tempranas, mediante la comparación de pares de imágenes, dos estadios distintos del desarrollo, de manera secuencial. Es decir, en el primer par de imágenes, se muestran embriones humanos de 1 y 2 días de desarrollo, en el siguiente par, embriones de 2 y 3 días, y así sucesivamente, hasta una fase del desarrollo de relevancia biológica (quinto día después de la fecundación) y totalmente distinta de la inicial (Universidad de Nueva Gales del Sur [unsw], 2019). De igual manera, la comparación de pares de imágenes es útil para promover la comprensión de los cambios estructurales de los sistemas corporales, así como la regulación de dichos cambios, con fotografías obtenidas del sitio electrónico de la unsw de artículos de revistas indizadas, especializadas en desarrollo, o imágenes originales de producción personal (figura 3). Hasta este momento, el ejercicio se ha realizado sin discusión o participación por parte del equipo docente o de los estudiantes. Al inicio del ejercicio, se indica que la actividad a realizar requiere de anotar en sus cuadernos, tres diferencias y tres similitudes entre las dos imágenes de cada par.

Al final de la secuencia de pares, se presentan las siguientes preguntas detonadoras:

- ¿Qué sucedió?

- ¿Qué procesos puedes decir que ocurrieron?

- ¿Qué estructuras puedes reconocer?

- ¿De qué manera cambiaron a lo largo del proceso?

Con estas preguntas, la intención es que hagan una reflexión respecto a los procesos generales y a nivel celular, que se encuentran involucrados en los cambios graduales durante el período del desarrollo expuesto. Los estudiantes hacen entonces una lista de los procesos, respecto a los cuales deberán hacer una búsqueda de información (en la bibliografía recomendada por el Departamento de Embriología o en sitios electrónicos como Wikipedia), para que en la siguiente sesión expongan sus hallazgos y los discutan con el resto del grupo. 
Izquierda. Johannes Vermeer. El arte de la pintura (Alegoría de la pintura), 1666. Museo de Historia del Arte de Viena.

Recuperada de: Google Arts \& Culture (s.f). Jan Vermeer - The Art of Painting - Google Art Project [fotografía].

Derecha. Pablo Picasso. L'Atelier (The Studio), 1927-1928. The Museum of Modern Art, New York.

Recuperada de: Tony Hisgett (2010). Pablo Picasso - The Studio [fotografía].

Figura 3. Ejemplos de una serie de pares de imágenes, para su comparación. En este caso, son fotomicrografías de cortes transversales de embrión de pollo, generadas con microscopía de fluorescencia (Hofmann, 2012, Instituto de Investigaciones Biomédicas, UNAM), en las que se muestran distintas etapas del desarrollo del tubo neural. En esta serie de pares de imágenes (que son presentadas a los estudiantes un par a la vez, de manera secuencial, 1, 2 y 3), la intención es que identifiquen por lo menos tres similitudes y tres diferencias entre la imagen $A$ y la imagen B de cada par. Con este ejercicio de comparación, los estudiantes podrán observar los cambios en la organización, la complejidad y la formación de las estructuras definitivas de la médula espinal, desde la etapa del desarrollo más temprana (1A) hasta la más tardía (3B).

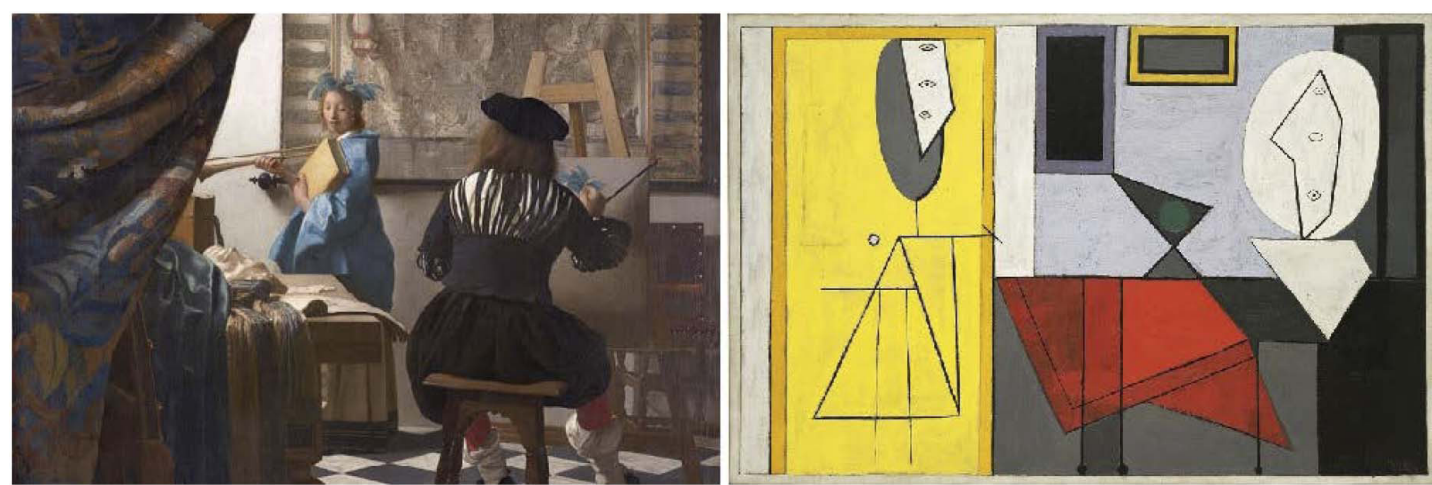

En etapas posteriores del cursoy con el objetivo de exponer de manera introductoria la relevancia clínica de los conceptos relativos a procesos del desarrollo, se utiliza nuevamente la estrategia de la comparación de imágenes, en los temas que, por su naturaleza, así lo permiten. En estos casos, las comparaciones se realizan entre imágenes (ultrasonografía, ultrasonografía Doppler, rayos- $X$, tomografía axial computarizada, resonancia magnética, resonancia magnética funcional, etcétera) de individuos normales y casos clínicos de malformaciones congénitas. A partir de la comparación de las imágenes mostradas en el par (sano vs. condición clínica), se formulan las preguntas que promueven la observación, identificación de estructuras y el descubrimiento de la malformación representada:

- ¿Qué sucedió?

- ¿Cómo se llama la condición?

- ¿Qué proceso del desarrollo ocurrió inadecuadamente?

- ¿Qué factores pudieron causar la condición observada?

- ¿En qué otros órganos o sistemas se podrían encontrar alteraciones por fallas en el mismo proceso del desarrollo?

Con los ejercicios de comparación de imágenes, se promueve, además de la observación en sí, la búsqueda de información relevante, así como la integración de los conceptos propios del desarrollo humano con su relevancia en el ámbito clínico. 
Aunque la incorporación del arte visual ha sido una de las estrategias con las que he experimentado, como resultado de la reflexión, ha hecho falta evaluar la eficacia en el logro de las bondades descritas por distintos autores, con el uso de estas dinámicas de enseñanza en los grupos de estudiantes a mi cargo. Para verificar que, mediante la implementación del método arriba descrito, se puede lograr el desarrollo de habilidades para la obtención de información visual o gráfica, la integración de datos, el razonamiento crítico y la aplicación de los conceptos en la solución de problemas propios del contexto profesional, es necesario diseñar instrumentos de evaluación pertinentes para llevar a cabo la comparación con grupos de estudiantes que hayan revisado los contenidos de la asignatura de una manera tradicional.

En conclusión, la incorporación de arte visual como elemento importante en la formación de futuros médicos ha resultado motivante para que los estudiantes identifiquen los cambios en la forma de los productos humanos durante el desarrollo intrauterino, infieran los procesos por los cuales suceden los cambios morfológicos y el crecimiento, aborden los contenidos planteados para la asignatura y realicen las búsquedas de información relevante respecto a las condiciones clínicas presentadas.

Para poder afirmar que la dinámica utilizada en el aula tiene una repercusión en la construcción de competencias profesionales, es necesario hacer una evaluación comparativa de las habilidades de identificar problemas a partir de la observación y de aplicar el conocimiento de los procesos del desarrollo dentro de contextos clínicos propios del ejercicio profesional del médico general.

Aunque en el panorama presentado en este artículo no se ha hecho una valoración del grado de desarrollo de conocimientos y habilidades en los estudiantes, el simple hecho de generarles la motivación por el abordaje de los conceptos que subyacen al desarrollo humano, dentro del campo de la medicina, es razón para continuar experimentando con estrategias de enseñanza que favorezcan el aprendizaje activo y significativo. Considero también, de suma importancia, que mi papel como docente en el aula no sea únicamente el de una estación de retransmisión de información entre los textos básicos y los estudiantes, sino el de guía para la construcción del conocimiento y la identificación de su relevancia en el ejercicio de la profesión.

\section{Referencias}

- Association of American Medical Colleges - Howard Hughes Medical Institute [AAmcHHMI] Committee. (2009). Scientific Foundations for Future Physicians. Association of American Medical Colleges \& Howard Hughes Medical Institute. Recuperado de: http://www.physics.emory.edu/faculty/weeks//journal/AAMC-HHMI.pdf. 
* Cooke, M., Irby, D. M., Sullivan, W. y Ludmerer, K. M. (2006). American Medical Education 100 Years after the Flexner Report. N Eng/ J Med., 355(13):1339-1344.

- De anatomische les van Dr. Frederick Ruysch [fotografía]. (s.f). Recuperada de: https:// commons.wikimedia.org/wiki/File:De anatomische les van Dr. Frederick Ruysch.jpg.

* Dolev, J. C., Friedlaender, L. K. y Braverman, I. M. (2001). Use of Fine Art to Enhance Visual Diagnostic Skills. JAMA, 286(9):1020-1021.

* Ferry, A. L., Hespos, S. J. y Gentner, D. (2015). Prelinguistic Relational Concepts: investigating analogical processing in infants. Child Dev., 86(5):1386-1405.

* Flexner, A. (1910). Medical Education in the United States and Canada: a report to the Carnegie Foundation for the Advancement of Teaching. Bulletin No. 4. B. D. Updyke. Boston: Merrymount Press.

* Flexner, A. (1925). Medical Education: a comparative study. New York: The Macmillan Company.

* Gentner, D. (2010). Bootstrapping the mind: analogical processes and symbol systems. Cogn Sci., 234(5):752-775.

* Goldwater, M. B. y Gentner, D. (2015). On the Acquisition of Abstract Knowledge: structural alignment and explication in learning causal system categories. Cognition, 137, 137-153.

* Google Arts \& Culture (s.f). Jan Vermeer - The Art of Painting - Google Art Project [fotografía]. Recuperada de: https://commons.wikimedia.org/wiki/File:Jan Vermeer - The Art of Painting - Google Art Project.jpg.

* Tony Hisgett (2010). Pablo Picasso - The Studio [fotografía]. Recuperada de: https://bit.ly/34stL1X.

* Jones, D. S. (2014). A Complete Medical Education Includes the Arts and Humanities. Virtual Mentor, 16(8):636-641.

* Kurtz, K. J., Boukrina, O. y Gentner, D. (2013). Comparison Promotes Learning and Transfer of Relational Categories. J Exp Psychol Learn Mem Cogn., 39(4): 1303-1310.

* Mauritshuis online catalogue (s.f). Rembrandt - The Anatomy Lesson of Dr Nicolaes Tulp [fotografía]. Recuperada de: https://commons.wikimedia.org/wiki/ File:Rembrandt - The Anatomy Lesson of Dr Nicolaes Tulp.jpg.

* Mazieres, Jean Louis (2016). IMG_5758 Joan Miro. 1893-1983. Femme et oiseaux au lever du soleil. Woman and Birds at sunrise. 1946. Fondation Joan Miro. Barcelone [fotografía]. Recuperada de: https://www.flickr.com/photos/mazanto/3185851136.

* Nico_ (2011). Salvador Dalí: Rostro del gran masturbador [fotografía]. Recuperada de: https://www.flickr.com/photos/nico /6887018056/in/photostream/.

* Reproduction Gallery (s.f). The Garden 1925 Painting By Joan Miro [fotografía]. Recuperado de: https://www.reproduction-gallery.com/oil-painting/1516516020/ the-garden-1925-by-joan-mirol. 
* Shapiro, J. y Shallit, J. (2014). A Night at the Museum—Helping Residents 'See' Their Patients. Virtual Mentor, 16(8): 599-603.

* Spencer, A. L., Brosenitsch, T., Levine, A. S. y Kanter, S. L. (2008). Back to the Basic Sciences: an innovative approach to teaching senior medical students how best to integrate basic science and clinical medicine. Acad Med., 83(7): 662-669.

* Universidad de Nueva Gales del Sur, Australia. Hill, M. A. (2019) Embryology Main Page. Recuperado de: https://embryology.med.unsw.edu.aul.

\section{Material adicional}

- Facultad de Medicina. (2010). Mapa Curricular, Licenciatura de Médico Cirujano. Universidad Nacional Autónoma de México. Recuperado de: http://www.facmed. unam.mx/fm/pal.

\section{Cómo citar este artículo}

* Hofmann Salcedo, Pablo (2019). Observación: la importancia del arte en la medicina. Revista Digital Universitaria (RDU). Vol. 20, núm. 6 noviembre-diciembre. Dol: http://doi.org/10.22201/codeic.16076079e.2019.v20n6.a7.

Recepción: 29/07/2019. Aprobación: 16/10/2019 